\title{
INJECTIVE MODULES AND CLASSICAL LOCALIZATION IN NOETHERIAN RINGS
}

\author{
BY ARUN VINAYAK JATEGAONKAR \\ Communicated by Alex Rosenberg, July 21, 1972
}

One of the main problems in the growing theory of noncommutative Noetherian rings can be loosely stated thus: If $\mathfrak{p}$ is a prime ideal of a Noetherian ring $R$, what should one mean by the localization $R_{\mathfrak{p}}$ of $R$ at $\mathfrak{p}$ ? When does $R_{\mathfrak{p}}$ exist and when is it nice? This problem has been considered by Goldie [1] and by Lambek and Michler [5]. In this note, we indicate a new approach to this problem and some of its advantages. We also introduce the concept of a left exact biradical for a ring, which may be of independent interest. Details will appear elsewhere.

As usual, a ring is Noetherian if it has the ascending chain condition on right ideals as well as left ideals. A subset of a ring is an Ore set if it is right Ore as well as left Ore. We refer the reader to [9] for all unexplained terminology and results concerning left exact radicals.

Let $R$ be a ring. The complete lattice of all left exact radicals for mod- $R$ (resp. $R$-mod) is denoted as $\boldsymbol{K}_{\boldsymbol{r}}$ (resp. $\boldsymbol{K}_{l}$ ). If $\mathscr{D}$ is a multiplicatively closed subset of $R, \rho_{\mathscr{D}} \in \boldsymbol{K}_{r}$ and $\lambda_{\mathscr{D}} \in \boldsymbol{K}_{l}$ are defined as follows: For each $M \in \bmod -R$ (resp. $M \in R$-mod), $\rho_{\mathscr{D}}(M)$ (resp. $\lambda_{\mathscr{D}}(M)$ ) is the largest submodule of $M$, each element of which is annihilated by some element of $\mathscr{D}$. If $\mathfrak{a}$ is an ideal of $R$, we define $\rho_{a}^{\#}$ as $\sup \left\{\rho \in K_{r} \mid \rho(R / \mathfrak{a})=0\right\}$ and $\lambda_{a}^{\#}$ as $\sup \left\{\lambda \in K_{l} \mid \lambda(R / \mathfrak{a})=0\right\}$. The multiplicatively closed set $\{r \in R \mid[r+\mathfrak{a}]$ is regular in $R / \mathfrak{a}\}$ is denoted as $\mathscr{C}(\mathfrak{a})$.

THEOREM 1 (cf. [5]). If $\mathfrak{s}$ is a semiprime ideal in a right Noetherian ring then $\rho_{\mathfrak{s}}^{\#}=\rho_{\mathscr{C}(\mathfrak{s})}$.

Matlis [6] has used localization to show that injective modules over a commutative Noetherian ring are nice. In the following two theorems, we establish an intimate connection between localizability and niceness of certain right injectives over a right Noetherian ring. Also see Theorems 7 and 8.

THEOREM 2. Let $\mathfrak{s}$ be a semiprime ideal in a right Noetherian ring $R$. Then the following four conditions are equivalent:

AMS (MOS) subject classifications (1970). Primary 16A08, 16A46, 16A52; Secondary $18 \mathrm{E} 40$.

Key words and phrases. Injective module, torsion theory, left exact radical, biradical, localization, classical localization, Ore set, Noetherian ring, stable semiprime ideal, localization of a Noetherian ring at a prime ideal, fully bounded Noetherian ring, Noetherian ring of Krull dimension one. 
(1) $\mathscr{C}(\mathfrak{s})$ is a right Ore set in $R$.

(2) There exists a right Ore set $\mathscr{C}$ in $R$ such that $\rho_{\mathscr{C}}=\rho_{\mathrm{s}}^{\#}$.

(3) Let $\mathscr{D}$ be any multiplicatively closed subset of $R$ such that $\mathscr{D} \subseteq \mathscr{C}(\mathfrak{s})$ and $\rho_{\mathscr{D}}=\rho_{\mathfrak{s}}^{\#}$. Then $\mathscr{D}$ is right Ore in $R$.

(4) Let $N$ be any right $R$-module which is $R$-isomorphic with a uniform right ideal of the ring $R / \mathfrak{s}$. Let $M_{R}$ be any essential extension of $N_{R}$ such that $M / N$ is $\rho_{\mathrm{s}}^{\#}$-torsion. Then $\mathfrak{s} \subseteq$ ann $M$.

THEOREM 3. Let $\mathfrak{s}$ be a semiprime ideal in a right Noetherian ring $R$ and let $\bar{R}=R / \rho_{\mathfrak{s}}^{\#}(R)$. Assume that $\mathscr{C}(\mathfrak{s})$ is a right Ore set in $R$. Then,

(1) $\rho_{\mathfrak{s}}^{\#}(R) \subseteq \mathfrak{s}$ and $\overline{\mathfrak{s}}=\mathfrak{s} / \rho_{\mathfrak{s}}^{\#}(R)$ is a semiprime ideal in the right Noetherian ring $\bar{R}$. The image of $\mathscr{C}(\mathfrak{s})$ in $\bar{R}$ is $\mathscr{C}(\overline{\mathfrak{s}})$ which is a right Ore set of regular elements of $\bar{R}$. If $R$ is a semiprime ring, so is $\bar{R}$.

(2) Let $R_{\mathfrak{s}}$ denote the classical right quotient ring of $\bar{R}$ with respect to $\mathscr{C}(\overline{\mathfrak{s}})$. Then $R_{\mathfrak{s}}$ is a semilocal right Noetherian ring with $J\left(R_{\mathfrak{s}}\right)=\overline{\mathfrak{s}} R_{\mathfrak{s}}$. The classical total right quotient ring of $\bar{R} / \overline{\mathfrak{s}}$ is isomorphic with $R_{\mathfrak{s}} / J\left(R_{\mathfrak{s}}\right)$.

(3) The injective hull of $R / \mathfrak{s}$ in mod- $R$ is $R$-isomorphic with the injective hull of $\bar{R} / \overline{\mathfrak{s}}$ in mod- $\bar{R}$ which, in turn, is $R$-isomorphic with the injective hull of $R_{\mathfrak{s}} / J\left(R_{\mathfrak{s}}\right)$ in $\bmod -R_{\mathfrak{s}}$.

The following example suggests that, in an attempt to localize a Noetherian ring $R$ at a prime ideal $\mathfrak{p}$, one should not overemphasize the set $\mathscr{C}(\mathfrak{p})$. Let $n>1$ be a positive integer and let $R$ be the subring of $M_{n}(Z)$, consisting of all those matrices in which all the entries below the main diagonal belong to $2 Z$. Let $\mathfrak{p}_{i}, 1 \leqq i \leqq n$, be the maximal ideal of $R$ consisting of all those matrices in which the $(i, i)$ th entry belongs to $2 \boldsymbol{Z}$. One can easily see that, in mod- $R$, the sequence

$$
0 \rightarrow \frac{\mathfrak{p}_{i} \cap \mathfrak{p}_{i+1}}{\mathfrak{p}_{i} \mathfrak{p}_{i+1}} \rightarrow \frac{\mathfrak{p}_{i+1}}{\mathfrak{p}_{i} \mathfrak{p}_{i+1}} \rightarrow \frac{\mathfrak{p}_{i+1}}{\mathfrak{p}_{i} \cap \mathfrak{p}_{i+1}} \rightarrow 0
$$

is exact and nonsplit and that

$$
\frac{\mathfrak{p}_{i} \cap \mathfrak{p}_{i+1}}{\mathfrak{p}_{i} \mathfrak{p}_{i+1}} \cong \frac{R}{\mathfrak{p}_{i+1}}, \quad \frac{\mathfrak{p}_{i+1}}{\mathfrak{p}_{i} \cap \mathfrak{p}_{i+1}} \cong \frac{R}{\mathfrak{p}_{i}},
$$

the indexing being modulo $n$. What should the localization of $R$ at $\mathfrak{p}_{1}$ be? In view of condition (4) of Theorem 2 , the sequence $(*)$ with $i=n$ indicates a "tie" of $\mathfrak{p}_{1}$ with $\mathfrak{p}_{n}$ and this obvious obstacle prevents $\mathscr{C}\left(\mathfrak{p}_{1}\right)$ from being a right Ore set in $R$. Condition (4) of Theorem 2 also suggests a remedy viz., try $\mathscr{C}\left(\mathfrak{p}_{1} \cap \mathfrak{p}_{n}\right)$. However, if $n>2$ then the sequence $(*)$ with $i=n-1$ indicates a tie of $\mathfrak{p}_{1}$ with $\mathfrak{p}_{n-1}$ via $\mathfrak{p}_{n}$ and this prevents $\mathscr{C}\left(\mathfrak{p}_{1} \cap \mathfrak{p}_{n}\right)$ from being a right Ore set in $R$. (Note: $\operatorname{Ext}_{R}^{1}\left(R / \mathfrak{p}_{n-1}, R / \mathfrak{p}_{1}\right)=(0)$.) In this way, one can see that if $\mathfrak{a}$ is any ideal of $R$ such that $\bigcap_{i=1}^{n} \mathfrak{p}_{i} \varsubsetneqq \mathfrak{a} \subseteq \mathfrak{p}_{1}$ then there is an obvious obstacle which prevents $\mathscr{C}(\mathfrak{a})$ from being a right or left 
Ore set in $R$. There is nothing obvious to prevent $\mathscr{C}=\mathscr{C}\left(\bigcap_{i=1}^{n} \mathfrak{p}_{i}\right)$ from being Ore. Indeed, it can be shown that $\mathscr{C}$ is an Ore set of regular elements of $R$ and that the localization of $R$ at $\mathscr{C}$ is the usual localization of the $Z$-order $R$ at the prime 2 in $Z$.

This example suggests that, given a prime ideal $p$ in a Noetherian ring $R$, one should seek a semiprime ideal $\gamma(\mathfrak{p})$ such that the associated prime ideals of $\gamma(\mathfrak{p})$ are precisely those prime ideals which have a "tie" with $\mathfrak{p}$ and then examine whether $\mathscr{C}(\gamma(\mathfrak{p}))$ is right Ore; if this set fails then $\mathfrak{p}$ is beyond first aid. In the context of HNP-rings with enough invertibles, a localization along these lines was developed by the present author [3]. Compared to the HNPR case, the "ties" between prime ideals in an arbitrary Noetherian ring are far from visible. To get an idea about these ties and get a candidate for $\gamma(\mathfrak{p})$, we have to introduce the notion of a "left exact biradical for a ring".

A left exact biradical for a ring $R$ is an ordered pair $(\lambda, \rho) \in \boldsymbol{K}_{l} \times \boldsymbol{K}_{r}$ such that $\lambda(R / t)=\rho(R / t)$ for every ideal $t$ of $R$. The partial order on the set $\boldsymbol{K}$ of all left exact biradicals for $R$ is defined by restricting the product partial order on $\boldsymbol{K}_{l} \times \boldsymbol{K}_{r}$. It turns out that $(\boldsymbol{K}, \leqq)$ is a complete lattice. If $\mathfrak{a}$ is an ideal of $R$, we define $\left(\lambda_{\mathfrak{a}}, \rho_{\mathfrak{a}}\right)$ as $\sup \{(\lambda, \rho) \in \boldsymbol{K} \mid \rho(R / \mathfrak{a})=0\}$. Clearly, $\rho_{\mathfrak{a}} \leqq \rho_{\mathfrak{a}}^{\#}$ and $\lambda_{\mathfrak{a}} \leqq \lambda_{\mathfrak{a}}^{\#}$; however, these inequalities may be strict. The particularly interesting case when $\mathfrak{a}=0$ will be dealt with elsewhere.

If $R$ is a commutative ring then there is an obvious bijection between $\boldsymbol{K}$ and $\boldsymbol{K}_{l}=\boldsymbol{K}_{r}$. If $R$ is a semiprimary ring then there is a bijection between $K$ and the set of central idempotents of $R$. If $\mathscr{D}$ is an Ore set in a Noetherian ring $R$, it can be shown that $\left(\lambda_{\mathscr{D}}, \rho_{\mathscr{D}}\right) \in \boldsymbol{K}$.

Henceforth, $R$ will denote a Noetherian ring, $P(R)$ will denote the set of all prime ideals of $R$ and $\mathfrak{s}$ will denote a semiprime ideal of $R$. Set $\Gamma_{0}(\mathfrak{s})=\left\{\mathfrak{p} \in \boldsymbol{P}(R) \mid \rho_{\mathfrak{s}}(R / \mathfrak{p})=0\right\}$. Let $\Gamma(\mathfrak{s})$ be the set of all those prime ideals of $R$ which are maximal in the set $\Gamma_{0}(\mathfrak{s})$. The set $\Gamma(\mathfrak{s})$ is our candidate for the set of all those prime ideals of $R$ which are "tied" to some prime ideal associated with $\mathfrak{s}$.

THEOREM 4. Let $\mathfrak{p}_{1}, \ldots, \mathfrak{p}_{n}$ be the prime ideals associated with a semiprime ideal $\mathfrak{s}$ of a Noetherian ring $R$. Let $\Gamma(\mathfrak{s}) \subseteq \Gamma \subseteq \Gamma_{0}(\mathfrak{s})$. Then $\Gamma_{0}(\mathfrak{s})=$ $\bigcup_{i=1}^{n} \Gamma_{0}\left(\mathfrak{p}_{i}\right), \Gamma(\mathfrak{s}) \subseteq \bigcup_{i=1}^{n} \Gamma\left(\mathfrak{p}_{i}\right)$ and $\rho_{\mathfrak{s}}=\inf _{1 \leqq i \leqq n} \rho_{\mathfrak{p}_{i}}=\inf \left\{\rho_{\mathfrak{p}}^{\#}: \mathfrak{p} \in \Gamma\right\}$.

With appropriate definitions, it can be shown that $\left(\lambda_{\mathfrak{s}}, \rho_{\mathrm{s}}\right)$ is a prime (resp. semiprime) in $\boldsymbol{K}$ if $\mathfrak{s}$ is a prime (resp. semiprime) ideal of $R$ (cf. [2]).

Let $\mathfrak{m}, \mathfrak{n} \in \boldsymbol{P}(R)$. We use the symbol $\mathfrak{m} \sim \mathfrak{n}$ to signify that there exist ideals $\mathfrak{a} \varsubsetneqq \mathfrak{b}$ in $R$ such that $\mathfrak{m b}+\mathfrak{b} \mathfrak{n} \subseteq \mathfrak{a}$ and $\mathfrak{b} / \mathfrak{a}$ is nonsingular in $(R / \mathfrak{m})$-mod as well as $\bmod -(R / \mathfrak{n})$. If there exists a finite sequence $\mathrm{m}_{1}, \ldots, \mathrm{m}_{k}$ such that, for $1 \leqq i \leqq k-1$, either $\mathfrak{m}_{i} \sim \mathfrak{m}_{i+1}$ or $\mathfrak{m}_{i+1} \sim \mathfrak{m}_{i}$ then we set $\mathfrak{m}_{1} \sim \mathfrak{m}_{k}$. If $\mathfrak{p} \in \boldsymbol{P}(R)$, let $\Omega(\mathfrak{p})=\{\mathfrak{q} \in \boldsymbol{P}(R) \mid \mathfrak{p} \sim \mathfrak{q}\}$. In several 
cases, it can be shown that $\Gamma(\mathfrak{p})=\Omega(\mathfrak{p})$. In general, we have

THEOREM 5. If $\mathfrak{p}$ is a prime ideal in a Noetherian ring $R$ then $\Gamma_{0}(\mathfrak{p})=$ $\bigcup\left\{\Omega(\mathfrak{m}): \mathfrak{m} \in \Gamma_{0}(\mathfrak{p})\right\}$.

If the set $\Gamma(\mathfrak{s})$ is finite, $\mathfrak{s}$ is called a nondegenerate semiprime ideal of $R$. For a nondegenerate $\mathfrak{s}$, we set $\gamma(\mathfrak{s})=\bigcap\{\mathfrak{p} \in \Gamma(\mathfrak{s})\}$. If $\Gamma(\mathfrak{s})$ is precisely the set of prime ideals associated with $\mathfrak{s}$ then $\mathfrak{s}$ is said to be a stable semiprime ideal of $R$. It can be shown that a semiprime ideal $\mathfrak{s}$ is stable iff $\rho_{\mathfrak{s}}=\rho_{\mathfrak{s}}^{\#}=$ $\rho_{\mathscr{C}(\mathfrak{s})}$ iff $\lambda_{\mathfrak{s}}=\lambda_{\mathfrak{s}}^{\#}=\lambda_{\mathscr{G}(\mathfrak{s})}$. In the example given above, $\gamma\left(\mathfrak{p}_{1}\right)=\bigcap_{i=1}^{n} \mathfrak{p}_{i}$ and it is stable.

We conjecture that all semiprime ideals in a Noetherian ring are nondegenerate and all but a finite number of them are stable.

THEOREM 6. Let $\mathfrak{s}$ be a nondegenerate semiprime ideal in a Noetherian ring $R$. Then $\gamma(\mathfrak{s})$ is a stable semiprime ideal of $R, \Gamma(\gamma(\mathfrak{s}))=\Gamma(\mathfrak{s})$ and $\left(\lambda_{\mathfrak{s}}, \rho_{\mathfrak{s}}\right)=\left(\lambda_{\gamma(\mathfrak{s})}, \rho_{\gamma(\mathfrak{s})}\right)=\left(\lambda_{\mathscr{C}_{\gamma(\mathfrak{s})}}, \rho_{\left.\mathscr{C}_{\gamma(\mathfrak{s})}\right)}\right)$ If $\mathfrak{a}$ is any stable semiprime ideal of $R$ such that $\left(\lambda_{\mathfrak{s}}, \rho_{\mathfrak{s}}\right)=\left(\lambda_{\mathfrak{a}}, \rho_{\mathfrak{a}}\right)$ then $\mathfrak{a}=\gamma(\mathfrak{s})$. If $\mathscr{D}$ is any Ore set in $R$ contained in $\mathscr{C}(\mathfrak{s})$ then $\mathscr{D} \subseteq \mathscr{C}(\gamma(\mathfrak{s}))$.

A nondegenerate semiprime ideal $\mathfrak{s}$ is said to be classical if $\mathscr{C}(\gamma(\mathfrak{s}))$ is an Ore set in $R$. Theorem 6 implies that if a prime ideal $\mathfrak{p}$ is classical in Goldie's sense [1] and if the intersection of the symbolic powers of $p$ is contained in $\rho_{\mathfrak{p}}(R)$ then $\mathfrak{p}$ is stable and classical in our sense.

Let $\mathfrak{s}$ be a nondegenerate semiprime ideal in a Noetherian ring $R$ and let $\mathscr{D}$ be a one-sided Ore set in $R$ such that $\mathscr{C}(\gamma(\mathfrak{s})) \subseteq \mathscr{D} \subseteq \mathscr{C}(\mathfrak{s})$. Is $\mathscr{D}$ necessarily a two-sided Ore set in $R$ ? The available information suggests that the answer should be in the affirmative.

We now indicate some applications of our approach to localization. Recall that a prime Noetherian ring is bounded if every essential onesided ideal contains a nonzero two-sided ideal. A Noetherian ring $R$ is fully bounded if $R / \mathfrak{p}$ is bounded for every $\mathfrak{p} \in \boldsymbol{P}(R)$. It is well known that a Noetherian ring $R$ is fully bounded if $R$ is finitely generated as a module over its centre; in such a ring $R$, it can be shown that every semiprime ideal is classical.

THEOREM 7. If $\mathfrak{s}$ is a nondegenerate semiprime ideal in a fully bounded Noetherian ring $R$ then $\mathfrak{s}$ is classical and the classical ring of quotients of $R$ with respect to the Ore set $\mathscr{C}(\gamma(\mathfrak{s}))$ is a semilocal fully bounded Noetherian ring.

THEOREM 8. Let $R$ be a fully bounded Noetherian ring. Then $\bigcap_{n=1}^{\infty} J^{n}(R)=$ (0). If $E$ is the injective hull of a simple right or left $R$-module then any finitely generated submodule of $E$ has finite length.

Assume that $R$ is semilocal as well. Let $m$ be a maximal ideal of $R$. Then 
$\Gamma(\mathfrak{m})$ consists of those maximal ideals $\mathfrak{n}$ of $R$ which have the following property: There exists a finite sequence $m=m_{1}, \ldots, m_{k}=n$ of maximal ideals of $R$ such that $\left(\mathrm{m}_{i} \mathrm{~m}_{i+1}\right) \cap\left(\mathrm{m}_{i+1} \mathrm{~m}_{i}\right) \neq \mathrm{m}_{i} \cap \mathrm{m}_{i+1}$ for $1 \leqq i \leqq k-1$. In particular, $\Gamma(\mathrm{m})=\Omega(\mathfrak{m})$.

Theorems 2, 3, 7 and 8 show that a substantial portion of the wellknown work of Matlis [6] on injectives over commutative Noetherian rings holds over fully bounded Noetherian rings. The finiteness assertions proved by Matlis can be obtained by imposing a suitable polynomial identity (cf. [8]).

Recall that a semiprime Noetherian ring $R$ has Krull dimension one iff $R / L$ is of finite length for every essential one-sided ideal $L$ of $R$ and $R$ is nonsemisimple.

THEOREM 9. Let $R$ be a semiprime Noetherian ring of Krull dimension one. If a semiprime ideal $\mathfrak{s}$ of $R$ contains an invertible ideal of $R$ then $\mathfrak{s}$ is classical and $\gamma(\mathfrak{s})$ is the prime radical of any invertible ideal of $R$ which is maximal among those contained in $\mathfrak{s}$. The classical quotient ring of $R$ with respect to $\mathscr{C}(\gamma(\mathfrak{s}))$ is a fully bounded semilocal semiprime Noetherian ring of Krull dimension one. Any right or left $R$-module $M$ of finite length can be uniquely decomposed as $M=K \oplus L$ where every composition factor of $K$ is annihilated by $\gamma(\mathfrak{s})$ and no composition factor of $L$ is annihilated by $\gamma(\mathfrak{s})$.

The above theorem shows that the usual localization of classical orders over commutative Dedekind domains [7] and the localization in HNPR developed in [3], [4] are special cases of our localization.

Let $R$ be a semiprime Noetherian ring with total quotient ring $Q$. Let $\mathfrak{s}$ be a semiprime ideal of $R$ such that $\rho_{\mathfrak{s}}(R)=(0)$; this condition is trivially satisfied if $R$ is a prime ring. The rings of quotients $Q_{\rho_{\mathrm{g}}}(R)$ and $Q_{\lambda_{\mathrm{g}}}(R)$ can be realized as subrings of $Q$. The subring $B_{\mathfrak{s}}(R)=Q_{\rho_{\mathfrak{s}}}(R) \cap Q_{\lambda_{\mathfrak{s}}}(R)$ of $Q$ may be an appropriate candidate for the localization of $R$ at $\mathfrak{s}$ even when $\mathfrak{s}$ is not classical. This construction can be generalized but, at present, we do not know whether the ring $B_{\mathrm{s}}(R)$ is of any interest in connection with $R$.

\section{REFERENCES}

1. A. W. Goldie, The structure of Noetherian rings, Lectures on Rings and Modules, Lecture Notes in Math., vol. 246, Springer-Verlag, Berlin, and New York, 1972.

2. O. Goldman, Rings and Modules of quotients, J. Algebra 13 (1969), 10-47. MR 39 \#6914.

3. A. V. Jategaonkar, An unpublished privately circulated letter to J. Kuzmanovich, November 1970.

4. J. Kuzmanovich, Localizution in HNP-rings (to appear).

5. J. Lambek and G. Michler, The torsion theory at a prime ideal in a right Noetherian ring (to appear).

6. E. Matlis, Injective modules over Noetherian rings, Pacific J. Math. 8 (1958), 511-528. MR 20 \# 5800. 
7. K. W. Roggenkamp and V. Huber-Dyson, Lattices over orders. I, Lecture Notes in Math., vol. 115, Springer-Verlag, Berlin and New York, 1970.

8. A. Rosenberg and D. Zelinsky, Finiteness of the injective hull, Math. Z. 70 (1958/59), 372-380. MR 22 \#12129.

9. B. Stenström, Rings and modules of quotients, Lecture Notes in Math., vol. 237, Springer-Verlag, Berlin and New York, 1971.

Department of Mathematics, Cornell University, Ithaca, New York 14850 (current address) 